\title{
The SOAR optical imager: status and first results
}

Hugo E. Schwarz, Michael C. Ashe, Maxime Boccas, Marco Bonati, Francisco Delgado, et al.

Hugo E. Schwarz, Michael C. Ashe, Maxime Boccas, Marco Bonati, Francisco Delgado, Ramon Gavez, Manuel Martinez, Patricio Schurter, Ricardo Schmidt, Roberto Tighe, Alistair R. Walker, "The SOAR optical imager: status and first results," Proc. SPIE 5492, Ground-based Instrumentation for Astronomy, (30 September 2004); doi: 10.1117/12.553185

Event: SPIE Astronomical Telescopes + Instrumentation, 2004, Glasgow, United Kingdom 


\title{
The SOAR Optical Imager: Status \& First Results.
}

\author{
Hugo E. Schwarz ${ }^{\mathrm{a}}$, Michael Ashe ${ }^{\mathrm{b}, \mathrm{c}}$, Maxime Boccas ${ }^{\mathrm{a}, \mathrm{d}}$, Marco Bonati ${ }^{\mathrm{a}, \mathrm{e}}$, Francisco Delgado ${ }^{\mathrm{a}}$, \\ Ramón Gálvez ${ }^{\mathrm{a}}$, Manuel Martínez ${ }^{\mathrm{a}}$, Patricio Schurter ${ }^{\mathrm{a}}$, Ricardo Schmidt ${ }^{\mathrm{a}}$, \\ Roberto Tighe $^{\mathrm{a}}$, Alistair Walker ${ }^{\mathrm{a}}$ \\ ${ }^{a}$ Cerro Tololo Inter-American Observatory, Casilla 603, La Serena, Chile ${ }^{*}$ \\ ${ }^{\mathrm{b}}$ Imaginatics Inc., USA \\ ${ }^{c}$ Southern Astrophysical Research (SOAR) Corp., \\ ${ }^{\mathrm{d}}$ Gemini Observatory, Chile, \\ eAstronomy Dept., Caltech, USA.
}

\begin{abstract}
We briefly describe the SOAR Optical Imager (SOI), the first light instrument for the $4.1 \mathrm{~m}$ SOuthern Astronomical Research (SOAR) telescope now being commissioned on Cerro Pachón in the mountains of northern Chile. The SOI has a mini-mosaic of $22 \mathrm{kx} 4 \mathrm{k}$ CCDs at its focal plane, a focal reducer camera, two filter cartridges, and a linear ADC. The instrument was designed to produce precision photometry and to fully exploit the expected superb image quality of the SOAR telescope over a 5.5x5.5 $\operatorname{arcmin}^{2}$ field with high throughput down to the atmospheric cut-off, and close reproduction of photometric pass-bands throughout 310-1050 nm. During early engineering runs in April 2004, we used the SOI to take images as part of the test program for the actively controlled primary mirror of the SOAR telescope, one of which we show in this paper. Taken just three months after the arrival of the optics in Chile, we show that the stellar images have the same diameter of 0.74 " as the simultaneously measured seeing disk at the time of observation. We call our image "Engineering $1^{\text {st }}$ Light" and in the near future expect to be able to produce images with diameters down to 0.3 " in the R band over a 5.5 ' field during about $20 \%$ of the observing time, using the tip-tilt adaptive corrector we are implementing.
\end{abstract}

Keywords: Instrumentation, imaging, CCDs, linear ADC

\section{INTRODUCTION TO SOAR}

The 4.1-m SOAR telescope was built on the northeastern spur of Cerro Pachón, Chile, at an altitude of 2700m, and a few hundred meters from the 8-m Gemini South telescope. SOAR itself has been discussed in detail elsewhere (and also in this volume) so in summary, it is a smaller version of the 8-m Gemini and VLT telescopes, with an actively controlled thin meniscus primary mirror. The telescope, it's dome, and facility are optimized to deliver the best possible images over a $\sim 10^{\prime}$ diameter field. The telescope is an f/16 Ritchey-Chrétien design for use over the wavelength range 300$2,500 \mathrm{~nm}$, with up to three instruments simultaneously mounted at each of the two Nasmyth platforms plus three more at bent Cassegrain stations, allowing nine "hot" instruments at any given time. In addition, the SOAR telescope has the capacity and interfaces needed to allow installation of (large!) Gemini facility instruments. Each focal station incorporates a fast CCD guider that drives the telescope tertiary mirror at up to $50 \mathrm{~Hz}$ in tip-tilt adaptive mode. A low altitude turbulence compensation instrument using Rayleigh laser guide stars -called the SOAR Adaptive module or SAM- is under design for the SOAR telescope at CTIO, and is described in another contribution to this conference. The telescope has seen engineering first light in March 2004, using the SOI instrument, and is being commissioned at the time of writing. The start of full science operations is planned for early 2005 . We follow Walker et al. ${ }^{2}$ in parts of this paper, and add the latest status, results, and detail any changes over the original design of the SOI. 


\section{SOI DESIGN PARAMETERS}

The principal SOAR image specification is that telescope and facility induced image blur should not exceed 0.18 " full width at half-maximum intensity (fwhm). This specification was derived from measurements of the $10^{\text {th }}$-percentile seeing on Cerro Pachon as 0.3 " fwhm, and the desire that the telescope plus facility should not degrade this figure by more than $10 \%$. Instruments are allowed a similar budget, i.e. the SOI is not allowed by itself to introduce more than 0.18 " of image blur, at zenith., over its full passband of 310-1050 nm. SOAR instruments are also required to exploit as much of the 10" diameter field as possible. The rationale for deciding the SOI instrument parameters was the complementary functions of the three large telescopes at the AURA Chilean observatory: the 4m Blanco on Tololo specializing in optical/NIR wide field observations (with up to $2^{\circ} \mathrm{FoV}$ ); Gemini south mainly optimized for the thermal IR, and SOAR to have medium field high image quality in the blue to NIR band.

- Top-Level requirements; achieved or implemented to date.

○ Image scale $0.08 \% /$ pixel; achieved $0.077 \% /$ pixel

○ Field of view 6x6'; implemented 5.3x5.3'

- Atmospheric Dispersion Corrector (ADC) to correct wavelengths longer than $320 \mathrm{~nm}$; implemented

○ At least six parfocal filters; implemented 10 positions, 8 mounted filters.

○ Instrument to produce less than 0.15 " image degradation at $550 \mathrm{~nm}$

- Additional requirements

O On-board tip-tilt sensor; implemented.

- Low scattered light; not fully tested yet, but initial results are good.

- Low heat leak; thermal IR camera images show no temperature increase when switched on.

○ At least 30 hours cryogen hold time; achieved $\sim 40 \mathrm{hrs}$

- Schedule requirement

- SOI to be first light instrument; done in March 2004

- SOI to be sited at a bent-Cassegrain port; implemented.

The SOI has now been built, commissioned, and partially tested on the telescope. The brief history of its birth is: Instrument concept approved by the SOAR Board in November 1998. The instrument passed CoDR in April 1999 and a pre-fabrication review in December 2000. It was delivered to SOAR in October 2003, and after the main telescope optics arrived during January 2004, the SOI was used to obtain $1^{\text {st }}$ light during March 2004 . The main difference from the original planned instrument is the installed CCD detectors. The foundry run Lincoln Labs chips were delayed to the extent that a rapid solution had to be found to replace these CCDs. The choice was to purchase two E2V 44-82 CCDs, which are now installed in the instrument. The completed SOI is shown in Figure 1, mounted on the bent-Cassegrain port of the SOAR telescope. 


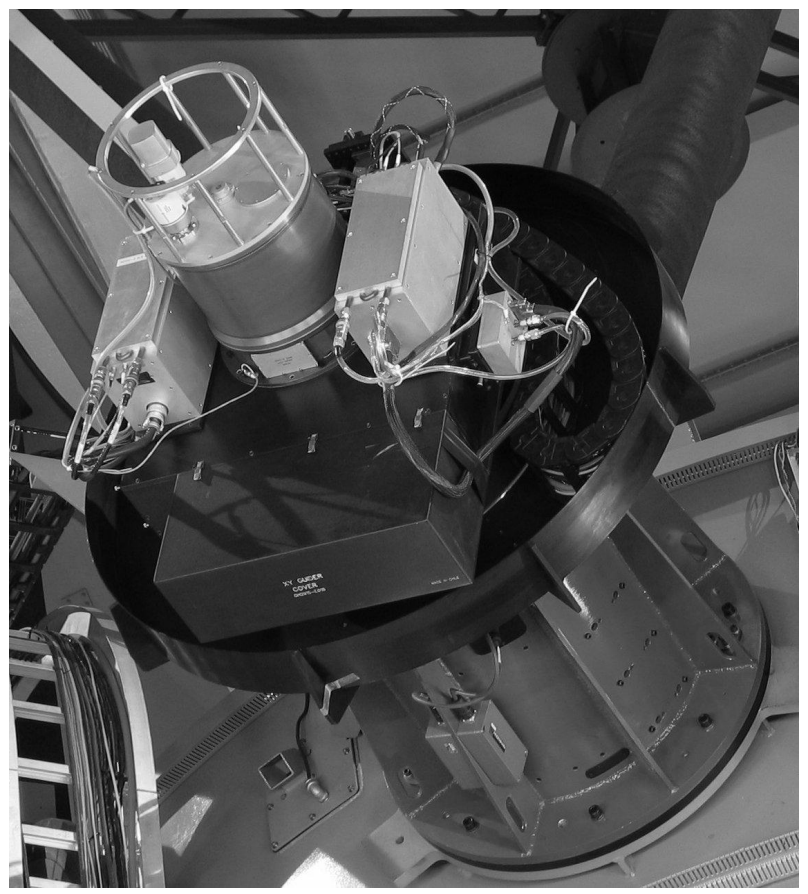

Figure 1: The SOAR Optical Imager mounted on the SOAR telescope bent-Cassegrain port. The large finned tubular part bolted to the telescope houses the two prisms of the atmospheric dispersion corrector (ADC), which at this focal position does not need to be rotated. At its lefthand end is the rotator, which supports the focal reducer optics, shutter box (square, below dewar), filter wheels, the cable wrap (large round, finned box), the main dewar with science CCDs (cylinder with "cage"), and the guide camera. There are two SDSS-2 (Leach) CCD controllers (rectangular boxes) mounted on the rotator on either side of the dewar. The SOI is mounted on the telescope using two precision locating pins so that the instrument is always easily positioned accurately with respect to the optical axis of the telescope after having been removed.

\section{OPTICAL DESIGN}

The SOAR bent Cassegrain position throws the telescope focus more than 2-m beyond the telescope structure, hence there is ample room to fit both an ADC and focal reducer optics in the converging f/16 beam. The two-prism linear ADC design adopted for the FORS instrument on the $\mathrm{VLT}^{3}$ is an attractive alternative to the traditional double pair of zero-deviation rotating prisms ${ }^{4}$., where space permits, and the optical input beam is relatively slow. To preserve throughput to $320 \mathrm{~nm}$ the rotating ADC design requires use of fused silica and $\mathrm{CaF} 2$ glasses, the latter is difficult to produce in the large diameter required, and is a delicate material to work with. No one to our knowledge has ever built such an ADC. We therefore chose the linear ADC design.

The linear 'trombone-like' ADC design is particularly suited for an alt-azimuth telescope as the angle between the image plane (i.e. the telescope altitude plane) and the atmospheric dispersion axis remains constant for all zenith distances, thus avoiding the need for prism rotation. This holds for any instrument that is --as is the SOI-- mounted on the telescope tube; only on a fixed Nasmyth platform would an ADC have to be rotated with the altitude axis. Our ADC consists of a pair of fused silica prisms with the same apex angle and a constant $180^{\circ}$ orientation offset. The forward prism corrects the atmospheric dispersion by moving longitudinally in the beam, and the fixed prism corrects the image plane tilt. An image shift occurs depending on the prism separation, this can easily be compensated for by the telescope pointing model. The focal reducer optical design is an adaptation of an original concept by Gilberto Morretto, which consists of a 6-element f/16 to f/9 reducer and field corrector, producing a global EE80 (80\% encircled energy) diameter $<0.31$ arcsec in each of the U, V, B, R, I broadband filters (typically 80-140 nm wide, 365-1000 nm center-wavelength range) over a flat $10 \times 10$ arcmin field. The original design was iterated in order to

- increase the back-focal distance to accommodate a tip-tilt guider pick-off

- include space for dual filter wheels and the dewar window

- decrease element thickness for ease of fabrication and improved UV transmission

- re-organize so that the single $\mathrm{CaF}_{2}$ element is encapsulated 
- include the linear ADC in the design

- relax the field size from $10 \times 10$ arcmin to $6 \times 6$ arcmin

The single image specification given in 1 . above was expanded to

- FWHM $<0.18$ " at zenith; equivalent to EE80 < 0.27 "

- FWHM $<0.34$ " at zenith distance $70^{\circ}$.; equivalent to EE80 < $0.51^{\prime}$

when imaging through astronomical broadband filters. The optical arrangement is shown in Figure 2 . Coatings on all exterior optics are Magnesium Fluoride over-coated in-house with solgel ${ }^{5}$, while the triplet is bonded using Corning Sylguard 184 transparent silicone. Theoretical throughput as a function of wavelength is shown in Figure 3, and compared with the measured transmission which is significantly better. The design met the image specification at all wavelengths, as an example through a broadband V filter $(500-600 \mathrm{~nm})$ the FWHM at zenith is 0.12 arcsec, and at zenith

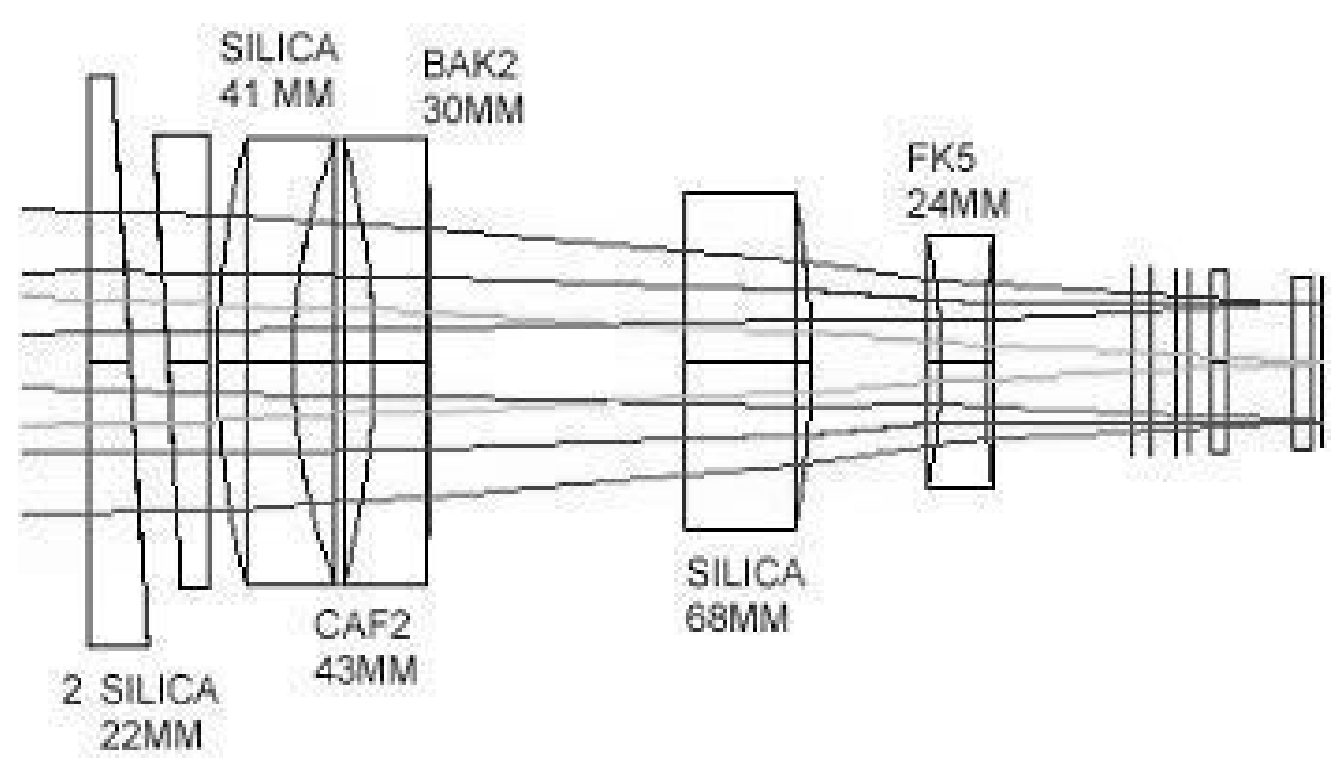

distance 70 degrees a FWHM of $0.29 \operatorname{arcsec}$ is achieved.

\begin{abstract}
Figure 2: SOI Optical Design, including ADC. From left to right are the two prisms of the ADC, shown in position corresponding to the telescope at zenith, then the cemented triplet, then two singlets. The two possible filter positions and the plane dewar window are indicated on the right. Labels indicate material and maximum thickness. Filters can be cemented combinations of Schott glasses, or interference filters. The dewar window is UV-grade fused silica, $10 \mathrm{~mm}$ thick.
\end{abstract}

The design was subjected to four additional analyses. The cemented triplet was subjected to a thermal analysis, considering a temperature variation of $-5 \mathrm{C}$ to $+20 \mathrm{C}$. For the fused silica-CaF2 interface, which is the most critical, we calculated that the compressive shear is entirely absorbed by the cement, and not in the glass. The maximum stress occurring in the cement is less than the shear elastic limit. The second test involved looked at the optical performance 
over the temperature range $-5 \mathrm{C}$ to $20 \mathrm{C}$ using Zemax; no image degradation occurs. Practical measurements have now determined the required thickness of the cement, and confirmed the theoretical results.
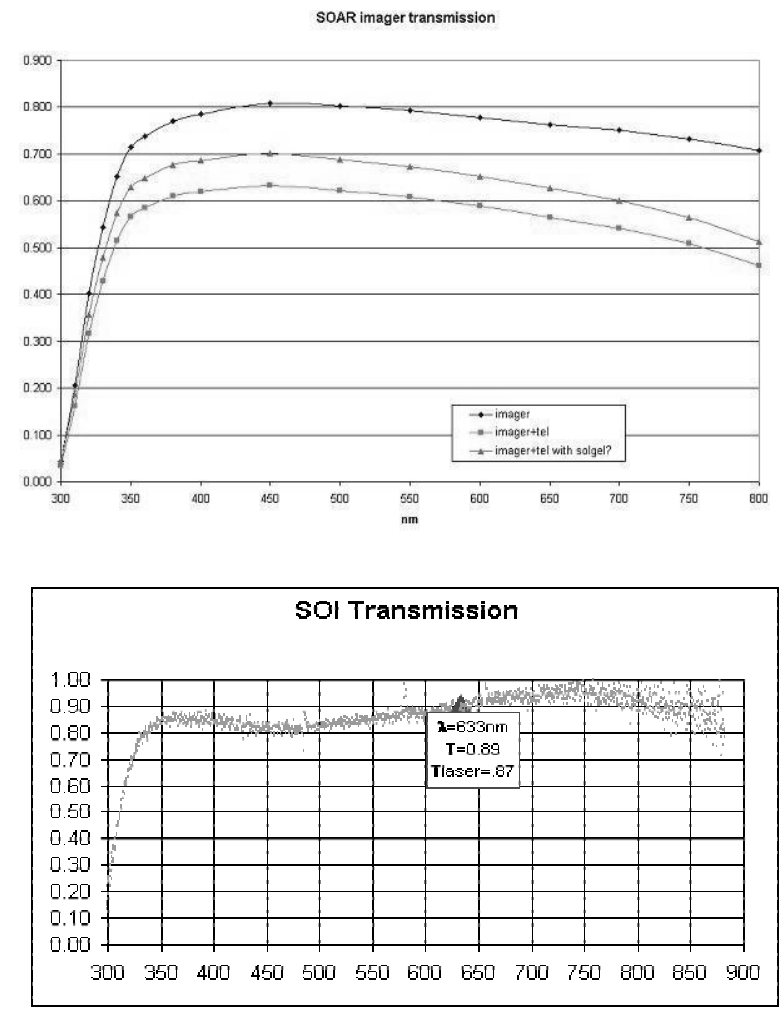

Figure 3: SOI throughput. Upper plot: The top trace shows the throughput of the imager optics with coatings as described in the text. The middle trace shows the effect of combining this with three mirrors, freshly-coated with aluminum. The lower trace shows the effect of not having Solgel coatings on the optics. Note these traces are calculated, not measured.

\section{Lower plot:}

The measured SOI instrument transmission. Note that over the range $330-800 \mathrm{~nm}$ the measured transmission exceeds the theoretical value of the upper curve above by up to $10 \%$. Below $350 \mathrm{~nm}$ the true curve is higher than shown because the equipment used suffered low efficiency in that range. The in-house solgel coating process has worked verv well.

We also performed a tolerance analysis and, as expected given the slow beam, there are no excessive mechanical demands on tilts, spacing, and centering. Finally a ghost analysis was performed; ghosts were simulated both by ray tracing and using the Zemax ghost focus generator. There is no "sky concentration" effect due to lack of any short radius of curvature element close to the focus. Considering the case of the reflection of incident light off the CCD surface, the worst case comes from the immediate reflection off the adjacent dewar window , $6 \mathrm{~mm}$ away. The ghost diameter is $760 \mu \mathrm{m}$. Assuming $40 \%$ of the incident light is reflected off the CCD and 3\% off the window, there is $1.2 \%$ of the incident light spread over 2016 pixels, thus producing a scattered light level of $6.10^{-6}$. We conclude that the behavior of the optics with respect to ghost images is benign. The usual precautions of using low reflectance paint, and trapping low angle-of-incidence reflections with annular stops, are taken to ensure that external light paths cannot reach the focal plane.

\section{INSTRUMENT DESIGN}

The overall mechanical design is constrained by the need to accommodate the bulky optical system, and the necessity of incorporating a rotator. The instrument weight limit is nominally $300 \mathrm{~kg}$, thus simple braced aluminum structures provide adequate strength and allowed us to only slightly exceed $(317 \mathrm{~kg})$ the weight budget. 
Since the ADC prisms do not rotate with respect to the telescope, the rotator need only support the focal reducer optics, shutter-filter-guider box and associated assemblies mounted thereon, and the dewar. The overall arrangement in shown in Figure 4. .

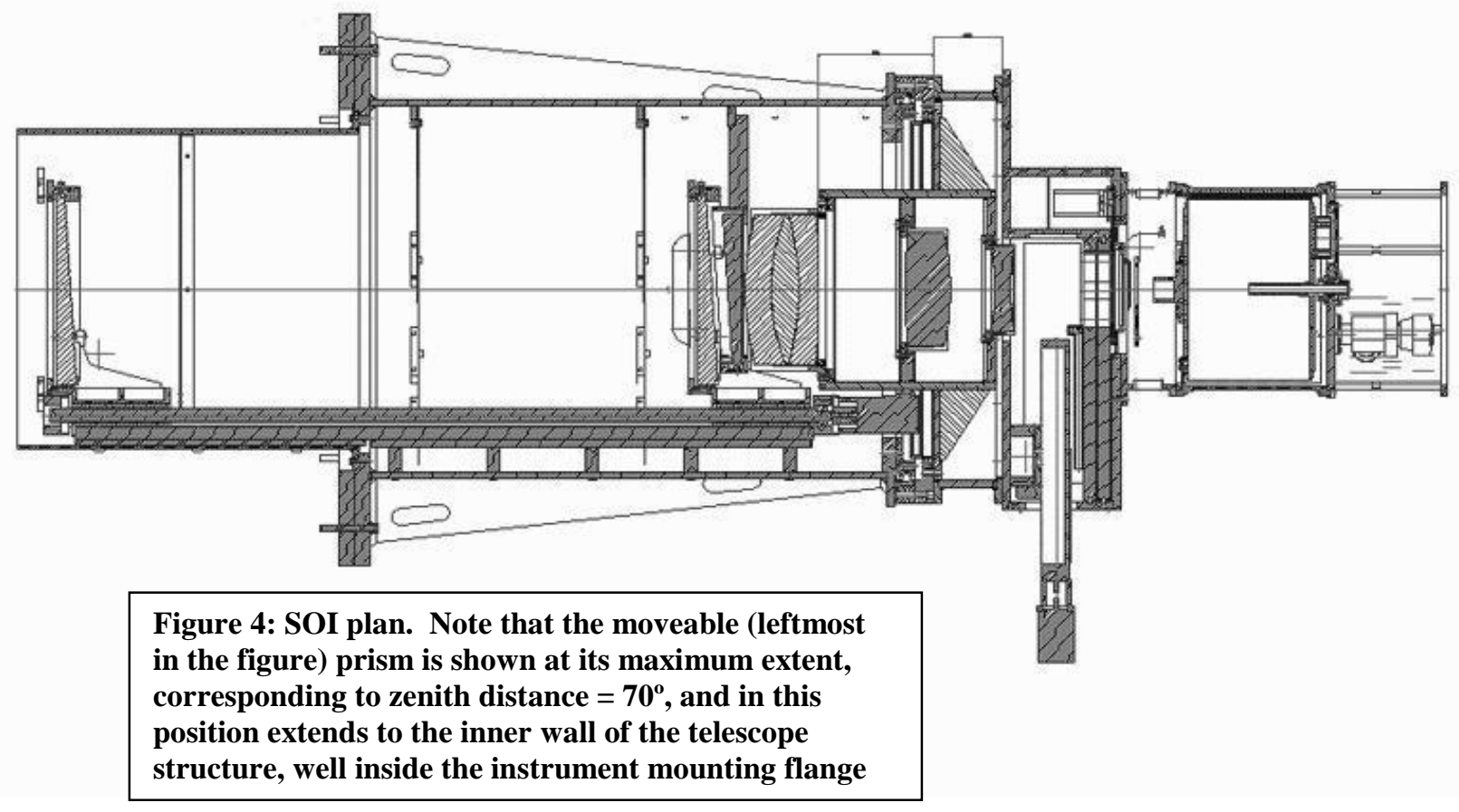

We have used Silvermax ${ }^{\mathrm{TM}}$ motors with success in several instrument projects. These "smart" servo motors, which have only a serial line and power connections, are easy to use, reliable, and have high integrity. We therefore decided to use these motors in the SOI, except for the filter wheels where we used heritage hardware.

The SOAR Project has adopted LabVIEW for telescope, dome and environment control and specified it for the instrument control as well. CTIO and the SOAR Project team developed ArcVIEW, a high-level LabVIEW interface for instrument control, and this will be used for the SOI and other SOAR facility instruments.

We now describe some of the sub-assemblies in more detail.:

- $\quad$ ADC assembly

The two prisms do not rotate with respect to the telescope and so their mounting system is attached to the large tubular assembly (Figure 1), which bolts to the telescope flange. One prism is fixed, the other is mounted on a Thompson linear stage, driven by a DC "smart" servo motor, controlled ultimately by the TCS, which feeds the instrument computer the zenith distance. The instrument computer then calculates the correct position to move the prism to in order to compensate for atmospheric refraction. We anticipate two modes of operation, the first is for the prism to be continuously tracking, the second is for the prism position to be set prior to an exposure, and then left in fixed position for the duration of the exposure. The stage motor is unavoidably close to the light beam, and so it is connected to a highconductivity strap to minimize creation of a hot-spot and possible air currents. 
- Focal reducer optics

All of the elements in the focal reducer are held in individual cells. The triplet is held by 8 preloaded spring elements, with hard points only in the axial direction. The compression of the spring elements was calculated to achieve an acceptable centering. The other two cells are of the "finger" type commonly used in CTIO instruments.

- $\quad$ Rotator and Cable-wrap

The rotator mechanism is driven by a servo motor (SilverMax(TM) QCI-23-3-E-01), directly coupled to a Harmonic Drive gear reduction, this turns a bronze friction wheel against a larger steel wheel, which houses a Kaydon 4-point ball bearing, which is attached to the rotating part of the instrument. Position sensing is achieved by a Heidenhahn ERA $780 \mathrm{C}$ tape encoder located inside the bearing housing. It has a resolution of 0.16 ", more than is needed. The rotator has a maximum rotation speed of $0.48 \mathrm{deg} / \mathrm{sec}$ and has redundant limit switches for safety. The cable wrap consists of an IGUS energy chain system, specially manufactured for this application, and is lubricated with Teflon to reduce friction.

\section{- Tip-tilt guider}

The tip-tilt guider consists of a probe containing a miniature $(10 \mathrm{~mm}$ side) right angled prism which diverts a small portion of the input beam to a second prism, where beam relay optics send it to the tip-tilt guider detector. The optics were designed by $\mathrm{T}$. Ingerson. The prism size and probe arm cross-section are small, the latter has cross-section of only $5 \mathrm{~mm}$ ) since they may need to occult the science beam if a guide star is desired, or can only be found, close to the field center. The probe assembly is mounted on a Parker-Daedalus XY-stage, with $0.5 \mu \mathrm{m}$ resolution. It is also possible to fine-tune the focus independently from the telescope focus. The detector system is a thinned, quad-readout Marconi CCD-39, which is thermoelectrically cooled, and operated by an SDSU-3 controller in frame-transfer mode.

\section{- Shutter}

The shutter is a single blade "focal plane" type driven by a dc servomotor via a cogged belt. It is low profile ( $4.7 \mathrm{~mm})$ and high performance. Minimum exposure time is less than $200 \mathrm{~ms}$, and repeatability below $0.5 \mathrm{~ms}$. The drive profile is trapezoidal. The shutter has been subject to extensive lifetime tests without degradation.

\section{- Filter wheels}

These are slim modules that were used with the now-retired CTIO 4-m telescope prime focus imager. Each has five positions, with change time between adjacent positions taking one second. Positional repeatability is better than 10 microns. These units have shown to be highly reliable during many years.

\section{- Dewar}

The dewar has a significantly longer hold-time specification of $30 \mathrm{hrs}$ than those presently in use at CTIO. We exceeded this requirement by 10-20 hrs by building a variant of the proven CTIO dewar design, which uses liquid nitrogen $\left(\mathrm{LN}_{2}\right)$ as cryogen. The thermal balance was modeled in order to determine the needed capacity. The dewar contains a highly polished aluminum can (volume 11.6 liters) that be filled to 50\% capacity via an axial tube, which incorporates a "no ice" vent, designed by Roger Smith. A single passive radiation shield surrounds the can. All interior surfaces are highly polished, and we have seen no need to incorporate layers of super-insulation, which introduce large surface area that can trap contaminants all too easily. The $\mathrm{LN}_{2}$ can is connected to the CCD-mount area by a copper braid which has had its thermal resistance optimized. The CCD mount is a kinematic design, with a frame coupled to the dewar front face by G- 
10 stand-offs. The frame in turn carries the CCD mount and the heater, which is servoed to keep the CCD at constant temperature to within $0.2^{\circ} \mathrm{C}$.

Temperature telemetry is relayed from the CCD mount, the $\mathrm{LN}_{2}$ can, and the vent tube area, the latter gives rapid and accurate indication when the LN2 is nearing exhaustion, well before the CCD mount temperature begins to rise. We also monitor dewar vacuum performance, using a compact Pirani/Cold Cathode sensor and a Pressure Display Unit (PDU), both from Pfeiffer Vacuum. The output from the PDU is converted to digital format and sent via a serial line to the instrument computer. Figure 5 shows the two E2V 44-82 CCDs mounted in the laboratory clean room.

\section{DETECTOR, CONTROLLER, \& DATA HANDLING}

The delays in obtaining the Lincoln Labs CCD-20's from the consortium foundry run, have resulted in the purchase of a

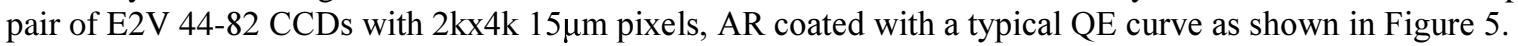
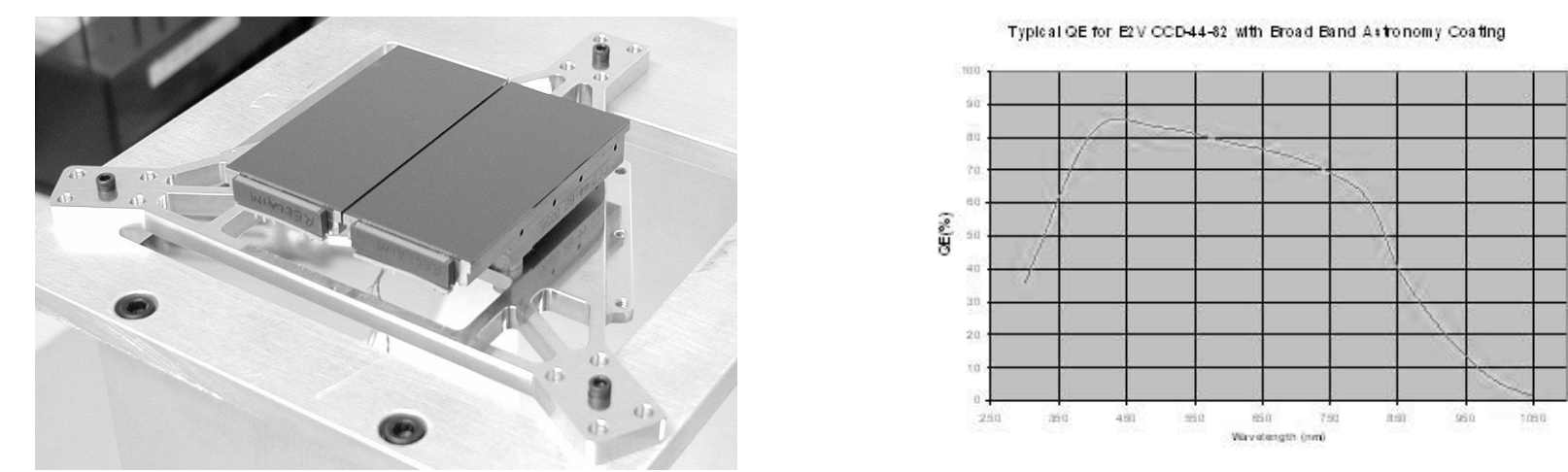

Figure 5: Left: Dual E2V 44-82 2kx4k CCDs mounted in the CTIO clean room before installation in the SOI dewar. These chips are butted with a gap of $0.55 \mathrm{~mm}$ and cover a field of 5.3 ' $x 5.3$ ' on the sky projecting 77mas onto each pixel. The mount is of $\mathrm{Al}$ alloy and astrometry is affected to the extent of $\sim 1 \mu \mathrm{m}$ per degree $\mathrm{C}$ over the full width. This corresponds to $5 \mathrm{mas} /$ degree $\mathrm{C}$. Across the gap this is 100 times smaller, or 50 $\mu \mathrm{as}$, and can be neglected over the temperature range the CCD is exposed to of less than 1 degree C. Right: Typical QE curve for the E2V 44-82 CCD used in the SOI. The QE is above 60\% between 350 and $810 \mathrm{~nm}$.

The CCDs are read-out using a SDSU-2 "Leach" detector controller, connected to a PC running Linux through a PCI board in a PCI-Linux-LabVIEW environment. The read-out time using two amplifiers per chip is $20 \mathrm{~s}$ at a RON of $4.5 \mathrm{e}$ (100s @ 2.5e). The same PC operates the instrument mechanisms except that the shutter is synchronized by the controller. The obtained data goes to another PC, but can also be sent directly to La Serena headquarters via a $155 \mathrm{Mbs}$ OC-2 microwave link or elsewhere via Internet II, currently with $10 \mathrm{Mbs}$, shared between Gemini, SOAR and CTIO.

The main goals of the software are to read the CCDs, write data to files, support various modes of operation such as onchip focusing of the SOI, scripting capability, motor, telescope and other systems communications, \& a user friendly GUI. The controller driver, other low-level routines, and several LabVIEW Virtual Instruments (V.I.'s) were developed in-house, while the ArcVIEW array control software was developed under contract by Imaginatics, Inc. The SOAR Communications Library (SCL) is a layer on top of LabVIEW and provides a reliable and flexible communications 
service between the components of ArcVIEW as well as with the other applications running in the same or in different machines throughout the network. ArcVIEW also provides scripting capability. ArcVIEW is described in detail elsewhere ${ }^{6,7}$ although still under development, particularly with respect to the final User GUI, it has proven powerful and efficient to use.

\section{STATUS AND $1^{\text {ST }}$ RESULTS}

At the time of writing, the SOI has had all its basic functionality confirmed. What is now left to do is the detailed characterization of the ADC, SOIs photometric and astrometric properties, the differential flexure between guide and science cameras, and the instruments long-term stability. The SOI has been used to obtain " 1 st engineering light" for the SOAR telescope, for which the main task now is the detailed control of the active primary mirror of the telescope, and during some of the initial tests, astronomical images were taken with the SOI.

In Figure 6, a Sloan r' band image of the globular cluster NGC6913 is shown. The exposure time was 30s, and no autoguiding was used. We also show the profile of a star from this image to have a FWHM diameter of 0.74", which is equal to the site seeing at the time of the observation, giving us some confidence that the SOAR plus SOI are not degrading the environmental seeing significantly. A 0.3 "additional term would increase the FWHM of the resulting image to 0.8 ", so we are confident that we are adding less than 0.3 " to the natural seeing.
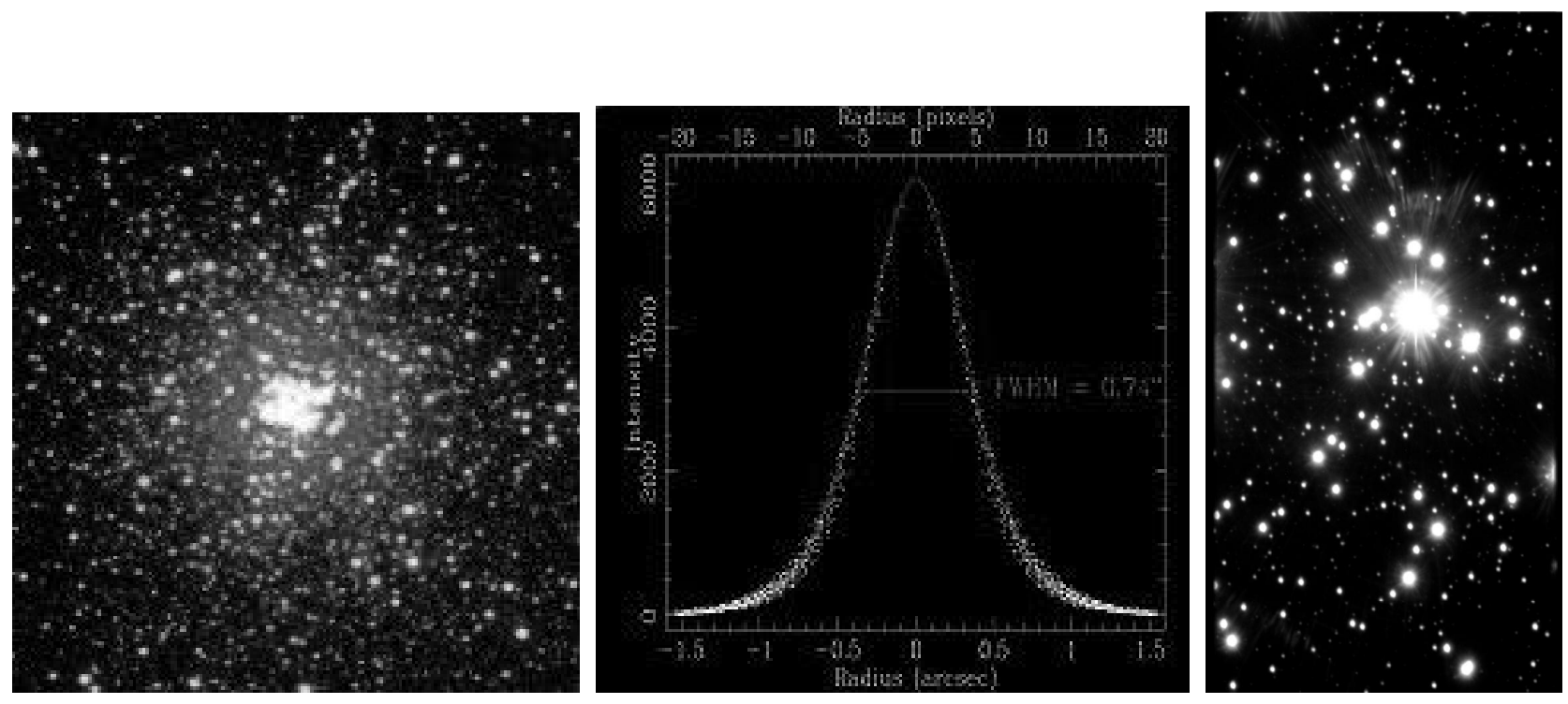

Figure 6: On the left, 1st engineering light image taken by the SOI through an SDSS $\mathbf{r}$ ' filter of the globular cluster NGC6913 on April 14 2004. Center, a plot of the image profile with a FWHM of 0.74". The local seeing monitor indicated a site seeing of about 0.8 'at the same time, indicating that the instrument did not degrade the image beyond the naturally imposed limit. On the right, a 10s exposure of the region around $\eta$ Carinae, with a seeing of 0.8 ". The striations visible near the brightest object in the field are due to the fact that the telescope had no primary or secondary baffles installed at the time of observation.

We expect to be able to take images with a quality of 0.4 " or better during about $20 \%$ of the available observing time, based on the seeing statistics of the Pachón area, once we have the telescope optics fully under active control, with proper baffling, and using an autoguider. We hope that SOAR will make major contributions to astrophysics over the years to come! 


\section{ACKNOWLEDGEMENTS}

We would like to thank Tom Ingerson, Roger Smith, and Gilberto Moretto for important contributions to the SOI design. Gerald Cecil and Steve Heathcote, in their respective capacities of SOAR Project Scientist and SOAR Director have provided sage advice. CTIO draftsmen, technicians and instrument makers are thanked for their skill and dedication, without which this instrument could not have been turned from design into reality.

NOAO is operated by AURA under cooperative agreement with the National Science Foundation (NSF). The Gemini Observatory is operated by AURA under a cooperative agreement with the NSF on behalf of the Gemini partnership: the NSF (United States), the Particle Physics and Astronomy Research Council (United Kingdom), the National Research Council (Canada), CONICYT (Chile), the Australian Research Council (Australia), CNPq (Brazil) and CONICET (Argentina).

\section{REFERENCES}

1. Sebring, T.E., Krabbendam, V.L., Heathcote, S., Wiecha, O., Schumacher, G. "SOAR Telescope Project: a progress report". Proc. SPIE 4837, 71, 2003

2. Walker, A.R. et al. Proc. "SPIE 4841, 286, 2003

3. Avila, G., Rupprecht, G., Beckers, J.M. “Atmospheric Dispersion Correction for the FORS Focal Reducers at the ESO VLT". Proc. SPIE 2871, 1135, 1997

4. Wynne, C.G., Worswick, S.P. "Atmospheric Dispersion Correctors at the Cassegrain focus”, MNRAS, 220, 657, 1986

5. Stilburn, J.R. "High efficiency sol-gel anti-reflection coatings for astronomical optics". Proc. SPIE 4008, 1361, 2000

6. http://www.qcontrol.com

7. Ashe, M.C., Bonati, M., Heathcote, S. "ArcVIEW: a LabVIEW-based astronomical instrument control system”. Proc. SPIE 4848, 508, 2003 